\title{
MÉDICOS-VIAJEROS Y VIAJEROS-CONVALECIENTES DE HABLA INGLESA EN LA ESPAÑA MEDITERRÁNEA DURANTE EL SIGLO XIX.
}

JosÉ RUIZ MAS

Universidad de Jaén

jrmas@ujaen.es

\section{RESUMEN}

Es mi intención en este artículo señalar el cambio de tendencia de los viajeros expatriados convalecientes de habla inglesa de residir en lugares tradicionalmente de moda como Italia, Francia y Portugal con anterioridad al siglo XIX por las costas mediterráneas españolas conforme avanzaba el siglo. Los primeros casos de residentes extranjeros que se instalaron en España fueron los acompañantes y parientes de los enfermos (hijos o esposas, como son los casos de Lord y Lady Holland y Richard Ford). El primer tercio del siglo XIX fue también testigo de cómo algunos de estos viajeros convalecientes, una vez instalados en España, se aprovecharon del caos reinante causado por las distintas desamortizaciones de propiedades eclesiásticas, especialmente de la de Mendizábal, para adquirir obras de arte españolas a precios muy ventajosos (Ford, Standish). A partir de mediados del siglo el cada vez mayor número de expatriados convalecientes residentes en España avanzó paralelamente a la exitosa publicación de relatos de viajeros médicos de habla inglesa por el Mediterráneo (Francis, Bennet, Lee) u otros viajeros ex-convalecientes (Cox, Playfair), todos los cuales hacían lo posible por recomendar en sus obras las zonas ideales de residencia según la dolencia del convaleciente. Estos relatos de viajes fueron pródigos a la hora de recomendar las costas, las ciudades costeras y las islas españolas (Baleares, Canarias) por su cálido clima y por la inexistencia de fiebres o epidemias. 


\section{ABSTRACT}

In this article I endeavour to portray how English-speaking invalid expatriate travellers changed their tendency to reside in traditional and fashionable places in Italy, France and Portugal prior to the 19th century and then they opted for Spanish Mediterranean coasts as the century advanced. The first cases of foreign residents in Spain were those who came accompanying their sick relatives (sons or wives, as were the cases of Lord and Lady Holland and Richard Ford). The first third of the 19th century saw how some of these invalid travellers, once in Spain, managed to take advantage of the chaos brought about by various Church land and property confiscations, especially those carried out by Mendizabal, to acquire Spanish works of art at very advantageous prices (Ford, Standish). From the middle of the century onwards, the increasingly higher number of invalid expatriates residing in Spain ran parallel to the successful publication of travel accounts in the Mediterranean by English-speaking medical-travellers (Francis, Bennet, Lee) or other ex-invalid travellers (Cox, Playfair), all of whom used to recommend the land to reside in according to the invalid's illness. In these travel books Spain's coasts and coastal towns as well as the islands (Balearics, Canary Islands) were usually highly recommended due to their warm climate and to their lack of fevers and disease.

Durante todo el siglo XIX tenemos constancia de la existencia de un considerable número de residentes de habla inglesa en España - y en general en el área mediterránea- que se habían instalado en nuestro país en busca de temperaturas más suaves como remedio o alivio a sus dolencias. Son los llamados invalids o viajeros-residentes enfermos y convalecientes, que empezaron a hacer acto de presencia por tierras españolas durante la primera mitad del siglo XIX. Para todos ellos era común la práctica del ocio y del reposo en sus lugares escogidos y disponer de suficientes medios económicos para una vida cómoda en España. Durante el siglo anterior los viajeros-convalecientes se habían decantado por Italia y Portugal.

Desde noviembre de 1802 a noviembre de 1804 Lord y Lady Holland viajaron por España por primera vez con la intención de proporcionar a su primogénito Charles un clima beneficioso para sus delicados pulmones y a su hijo pequeño Henry Edward espacios abiertos y baños de mar para un defecto congénito de rodilla. A pesar de que no se detuvieron durante mucho tiempo en ningún sitio de la geografía hispana, los niños fueron mejorando lentamente. Durante el viaje, Elizabeth Vassall Fox, más conocida como Lady Holland (1770-1845) — y más aún por su tiránico y agresivo carácter- redactó un diario, The Spanish Journal of Elizabeth Lady Holland, publicado en 1910 por el conde de Ilchester, que incluye información de primera mano sobre los personajes políticos españoles más relevantes del momento, así como lo más granado de la aristocracia de la época, inclusive la realeza, a cuyos miembros los Holland conocieron en un besamanos en Aranjuez. No puede decirse realmente que los Holland fueran auténticos expatriados, pero relatan muchos aspectos de la vida de los — aún relativamente escasos- residentes británicos en la España inmediatamente anterior a la Guerra de la Independencia. 
Durante el viaje de 1802 a 1804, cuando se instalaban en alguna ciudad, los Holland procuraban llevar una vida tan tranquila como les permitía la salud variable de los hijos. Lord Holland ocupaba su tiempo escribiendo una biografía y estudiando las obras de Lope de Vega y su esposa se dedicaba a leer (31). Asimismo cenaban y alternaban con los residentes británicos del lugar, los cuales también les proporcionaban con frecuencia alojamiento en sus casas o propiedades. Durante sus estancias en España, sobre todo en el primer viaje, el matrimonio Holland a menudo recibía y ofrecía alojamiento y entretenimiento a sus compatriotas: por ejemplo, en Sarriá (a tres quilómetros de Barcelona) un tal Mr Stembor les cedió su casa de campo o villa y ellos aceptaron encantados, sobre todo tras haber logrado encontrar sólo una triste mansión en una lúgubre calle, poco adecuada para la salud de su hijo Charles (1910: 7). En Valencia conocieron al cónsul británico, Mr Vague, y a dos ingleses apellidados Gordon, uno de los cuales viajaba por España por motivos de salud, por cierto muy precaria (26-27).

En su viaje por España los Holland fueron topándose con innumerables problemas para encontrar alojamiento adecuado para ellos, no sólo por su condición de exigentes aristócratas guardianes de su privilegiado estatus social, sino por razones de salubridad. Razona Lady Holland que sus dificultades para pernoctar y pasar temporadas como residente en las distintas ciudades visitadas se debía a que prácticamente nadie viajaba entonces por España con otros fines que no fueran los negocios, y siempre de paso. En consecuencia, las estancias algo más duraderas suponían para los viajeros en busca de la salud perdida un más que considerable gasto. Se queja la aristócrata de que «(...) The traveller who arrives for a couple of months in a town, must incur the same expense as if he were to remain ten years, - furnish and buy the furniture of a house from a joint stool to a spit» (19), gastos a los que, añade, no han de enfrentarse los españoles que viajan por su propio país, pues recurren al alojamiento en casas de parientes y amigos. Los Holland pronto aprendieron también a hacer uso de sus contactos e influencias entre sus compatriotas para encontrar dónde pasar alguna noche o dónde residir en las distintas ciudades españolas que fueron visitando. Resulta evidente por los datos que aportan que gran parte de los expatriados que residían en España a principios del siglo XIX eran sobre todo diplomáticos, comerciantes y banqueros y algún que otro militar contratado por el gobierno español, siendo la figura del viajero-convaleciente o invalid aún infrecuente.

El abogado inglés Richard Ford (1796-1858), considerado como el mayor entendido de los asuntos de la España de la época, el «Rerum Hispaniae indagator acerrimus» por excelencia del siglo XIX, fue residente en Sevilla desde 1830 a 1833, si bien pasó también algún que otro paréntesis veraniego en Granada entre tales fechas. Ford hizo el sacrificio de viajar a la imprevisible España para que su esposa Harriet pudiera gozar de un clima que beneficiase su frágil salud, mermada por tantos embarazos. En las obras de Ford, principalmente en la monumental A Handbook of Travellers in Spain, and Readers at Home (1845), en su continuación, Gatherings from Spain (1846), en sus reseñas literarias así como en su correspondencia con sus amigos, parecen coexistir el amor y la antipatía hacia España, los españoles y nuestro carácter. No puede por lo tanto decirse que Ford se trasladara a España por gusto. Según le reconocía a su amigo, el embajador plenipotenciario británico en Madrid Henry Unwin Addington (1790-1870), en carta de 15 de septiembre de 1830, él y su esposa estaban «(...) condemned to spend a winter or two in a warm climate» como el del sur de España (Prothero, 1905: 2). Sabemos que Ford lle- 
gó a plantearse residir en Málaga, ciudad que le recomendó su cónsul británico, Mr William Mark ${ }^{1}$, por ser ésta, en palabras del diplomático, «a second Paradise» (3). También se planteó Granada, calificada por su idolatrado duque de Wellington de «charming» (3). En una aún turbulenta España, según Alberich (2001: 82), la elección de Sevilla pudo estar condicionada, entre otros motivos, por su cercanía a Gibraltar, refugio de los británicos en caso de guerra civil en España, siempre tan a la vuelta de la esquina. Pero también es probable que para decantarse por Sevilla pesasen en él otros factores ajenos a la tranquilizadora cercanía al Peñón: de hecho, más cerca estaba Cádiz, que tan favorablemente le impactó en un principio y no fue sin embargo el lugar elegido. Quizás creyese Ford que Sevilla era la ciudad del sur de la Península donde podría llevar una vida social más intensa. $\mathrm{O}$ donde pudiera ejercer con más comodidad sus labores de información para su amigo el embajador Addington ${ }^{2}$.

Según relata Ford en el «Handbook», España no merecía ser visitada salvo por tres tipos de viajeros: para los interesados en estudiar la agreste y virginal naturaleza del país (1845:77); para los aficionados al arte y las antigüedades (especialmente los interesados en Murillo y Velázquez) (77); y para los que deseaban recrearse contemplando su arquitectura (78). Para todos ellos, asegura Ford, España es el país ideal. ¿YY los convalecientes? Para el Ford de los años cuarenta, época en que redactaba el «Handbook», la visita al país por motivos de salud no estaba entre las principales razones del viajero, si bien, resulta paradójico, fue éste el principal motivo que le trajo a Sevilla a principios de la década de los treinta. En la tercera edición del «Handbook» (1855) Ford dice ya ser plenamente consciente de las posibilidades de España, sobre todo de Andalucía, como lugar de residencia para los invalids venidos de allende nuestras fronteras. Ford describe además la suerte inmerecida de Andalucía, la región más favorecida de la piel de toro, ideal para los convalecientes extranjeros:

Andalucia is admirably suited to our invalids; here winter, in our catch-cold acceptation of the term, is unknown. The genial climate forms, indeed, one of the multitudinous boasts of the natives, who pride themselves on this «happy accident» thus lavished on them by nature, as if the bright skies were a making and merit of their own (1855: 127).

Para su grata sorpresa, no sólo encontró Sevilla barata, condición que le permitió llevar en ella una vida regalada por la mitad de lo que le hubiera costado en Londres, sino que consiguió que su compatriota Frank Hall Standish le alquilase una casa excepcional para el convaleciente en busca de la salud perdida. En carta fechada en 27 de septiembre de 1830 Ford se la describe a Addington en los siguientes términos: «It has the advantages of a garden, a fireplace, and a southern exposure, which make it perfectly warm» (Prothero, 1905:9). Una vez instalado, se encuentra ya preparado para empezar lo que él llama crípticamente la «gran caza» («grande chasse») (9). Con posterioridad, y gracias a

\footnotetext{
${ }^{1}$ William Mark (1782-1849) fue cónsul británico en Málaga desde 1824 a 1836. A partir de esta fecha le sustituyó en el cargo su hijo William Penrose Mark hasta su muerte, acaecida en 1872. Los posteriores representantes diplomáticos británicos en Málaga durante los años restantes del siglo XIX fueron Richard Wilkinson (desde 1873) y Alexander Finn.

${ }^{2}$ Para más información sobre la estancia de Ford en Sevilla y sus posibles actividades de espionaje, consúltese Ruiz Mas (2006).
} 
las gestiones del general irlandés O’Lawlor («don José»), administrador de las propiedades españolas de Wellington y también expatriado residente en Granada, Ford y su familia pudieron también disponer para los crudos veranos andaluces de los incómodos pero frescos y románticos aposentos del gobernador en la Alhambra (32), privilegiada residencia de la que ya disfrutara años antes, durante su estancia granadina a finales de la década de los veinte, su amigo el diplomático y escritor norteamericano Washington Irving (1783-1859). No fue Ford, como es de todos sabido, un convaleciente precisamente, pero sí lo era su esposa Harriet. Sin embargo, la estancia española del futuro hispanista resultó ser más que fructífera. Llegó a aprender bien nuestra lengua y a conocer nuestra historia y cultura, con gran profundidad en algunos aspectos, superficialmente otros, y con numerosos y evidentísimos prejuicios y fobias en muchos otros (Alberich, 2001: 87). Transcurridos tres años en España, regresó a Inglaterra en diciembre de 1833 para no volver más a poner pie en tierra hispana, mas no por falta de ganas.

Una vez aquí muchos invalids o sus acompañantes descubrieron - ya lo habían intuido algunos - una mina casi virginal de obras de arte y piezas de anticuario que, cuando su precaria salud se lo permitió, no dejaron escapar. Una situación caótica de cuidado y custodia del rico patrimonio artístico español durante y con posterioridad a la Guerra de la Independencia contribuyó a aumentar las ganancias materiales de estos convalecientes aficionados a la compra de obras de arte. Algunos de estos proto-invalids y sus acompañantes también dejaron constancia por escrito de sus experiencias e impresiones, aventuras y desventuras vividas u oídas en España, de ahí que sepamos lo que sabemos sobre ellos.

Ford, conocido en Sevilla como «Don Ricardo», de espíritu inquieto y siempre ávido de conocimiento y nuevas sensaciones, se dedicó a viajar extensamente por la Península, a dibujar paisajes, a tomar notas de sus vivencias españolas y a buscar obras de arte y libros de valor (la «grande chasse»), en una ocasión en compañía de su esposa, en otra con la única compañía de un criado; y, tal como sugiere Alberich (2001: 86), muy probablemente con los seis volúmenes de las guías artísticas de Ceán Bermúdez (Diccionario de las Bellas Artes, 1800) bajo el brazo. El Ribalta que Don Ricardo adquirió en Valencia nos demuestra la intención artístico-coleccionista del viajero inglés. No puedo evitar considerar más que probable que sus extensos viajes «turísticos» por gran parte de la geografía española tuvieran, al menos en parte, la inconfesada intención de buscar lienzos y otros objetos de arte. Otra prueba de sus reconocidos intereses artísticos durante su estancia española es el pasaporte usado en su primer viaje por tierras hispanas con su esposa en 1831. En el documento, expedido por el Conde de España, se conmina a todas las autoridades civiles y militares «whether with the pen or sword, to aid and assist the bearer in his examination of the fine arts and antiquities of the Peninsula» (Brinsley Ford, 1974: 60).

Ford no se fue del país de vacío. Cierto es que, en comparación con otros compatriotas y otros viajeros franceses, rivales todos en la adquisición de obras de arte españolas, sus logros fueron más modestos, mas tampoco desdeñables: dos Alonsos Canos, tres Zurbaranes, nueve Murillos, un Ribalta, un Greco y dos Velázquez (un retrato de Mariana de Austria), más otros lienzos de Herrera el Viejo (maestro de Velázquez), Herrera el Mozo, Juan Carreño de Miranda, Luis de Morales, Ignacio Iriarte, Pedro de Moya, Fernando Gallegos, etc., numerosos libros españoles de considerable valor, una extensa 
colección de monedas antiguas y una gran cantidad de auténticos fragmentos de azulejos de la Alambra, amén de otras piezas no identificadas (cuadros y joyas) que le dejaron al final de su estancia española - le contaba a su amigo Addington por vía epistolar- en una situación económica que hacía perentoria la práctica del ahorro (Prothero, 1905:26). Para 1836 Ford ya se había cansado de su colección de lienzos y se quiso desprender de la mayoría de ellos, de veintisiete para ser precisos ${ }^{3}$. En mayo de ese mismo año le escribía a Addington que ya no le producían placer, sino gastos y problemas. El placer se lo proporcionó el conseguirlos, no el poseerlos (Bean, 1995:17).

Analicemos también el curioso caso del citado Frank Hall Standish (1799-1840), viajero-invalid nada convencional que retrató la España posterior a la guerra de la Independencia. Éste se encontraba a mediados de 1835 de viaje por Turquía como parte de un viaje por las costas del Mediterráneo. Fruto de este periplo por España, Malta, Grecia, Italia y Turquía vieron la luz sus dos tomos de The Shores of the Mediterranean (1837 y 1838). España, el primero de los países visitados, le había dejado mal sabor de boca. Era, a su juicio, un país incivilizado, supersticioso, fanático, arruinado, decadente, envidioso, cruel, etc., entre otros calificativos poco generosos para con nuestras costumbres y carácter. Para Standish las únicas virtudes del país eran su clima benevolente, ideal para el enfermo de reumatismo y de gota —enfermedad ésta que a él aquejaba (I:xii-xiii)- sobre todo en ciudades como Cádiz y Málaga, y su riqueza artística, especialmente la de Sevilla, según el inglés sólo superada por Roma (I:10). Tras dos años de residencia en España a principios de la década de los treinta, su opinión de los españoles era radicalmente negativa, si nos atenemos a lo que también escribía en The Maid of Jaen, a Poem $(1832)^{4}$. A pesar de su antipatía por España, en 1836 Standish hizo de tripas corazón para volver a pasar por nuestro país durante su viaje por las costas mediterráneas. Volvió a recorrer los lugares de culto de las principales ciudades del sur español, sobre todo en Sevilla, para admirar los cuadros de nuestros maestros, mas poco pudo hacer en aras de la mejora de su colección particular de obras de arte, salvo sentir envidia por la del vicecónsul Williams, poseedor en 1832 de nada menos que treinta y siete Murillos (Alberich 1976:80). En efecto, esa necesidad de «intellectual gratifications» (1837, I: xiii) a las que decía Standish aspirar en sus viajes por el Mediterráneo como consecuencia de su pasión por las obras de arte, según relataba en el primer volumen de The Shores of the Mediterranean (1837), le volvieron a traer a toda prisa a la odiada España en febrero de 1836, mientras se encontraba visitando Constantinopla. En efecto, al tener Standish noticia de

\footnotetext{
${ }^{3}$ Gracias a las diez cartas de Ford a su agente artístico en Inglaterra, Dominic Colnaghi (1790-1879), encontradas por Thomas Bean en un ejemplar del «Handbook» que adquirió en una subasta, conocemos con cierto detalle la trayectoria de las adquisiciones artísticas del viajero inglés. Bean (1995) ofrece un recuento de las obras de pintores españoles que Ford fue comprando durante su estancia española y de las obras que terminó vendiendo en 1836.

${ }^{4}$ «Were I asked whether, from what I had seen of the present race of Spaniards, I should be disposed to like them, I should say certainly not; and my reason for saying so, would be the dislike I have to their arrogance, their prejudice against foreigners, and their general hypocrisy of character, and insolence and brutality when in office. This is the opinion I have drawn from a long experience of, and two years' residence amongst the Spaniards. Let anyone who differs from me, and has not been amongst them, frequent them and judge for himself. Let anyone who has not such opportunity, read Col. Napier's History of the Peninsular War, a work as honourable to the British army (being written by one of its officers) as any battle won, and he will, I think, not find me too severe in my remarks» (1832:58).
} 
la desamortización de Mendizábal, interrumpió su estancia en Turquía y le faltó tiempo para regresar a nuestros lares, ahora, eso sí, con una actitud más positiva hacia todo lo hispano (inclusive las corridas de toros, que tanto le disgustaban antes), a la caza despiadada de obras de arte:

Great changes have taken place in the political world of Spain since my departure; the friars have been driven from their convents, and their effects sold. All the paintings of the province [of Seville] are to be collected, and the best reserved to form a museum, the others are destined to the hammer (1838, II: 220).

Tanto el epistolario escrito por los visitantes y los residentes extranjeros como los relatos de viajes publicados por éstos mismos aportan relevante información sobre la vida de los cónsules británicos destinados en las ciudades de nuestra geografía en las que se había instalado alguna comunidad de residentes anglosajones. En una carta a Addington fechada en marzo de 1833, Ford se quejaba de la próxima llegada al país de nuevos cónsules, «a consular deluge», hecho demostrativo de la necesidad de atender a los cada vez más numerosos británicos en tierras españolas. A pesar de las críticas que les dedica Ford, no debe olvidarse que algunos de ellos contribuyeron no poco en la mejora de las condiciones sociales y espirituales de sus compatriotas en una España llena de prejuicios contra todo lo foráneo en asuntos religiosos. El propio Standish (1838, II: 321) consideraba al cónsul de Málaga Mr William Mark una persona dinámica y eficiente, entre otros motivos, porque había logrado arrancar de las autoridades españolas la construcción de un cementerio protestante a las afueras de la ciudad, recinto que el desafortunado teniente Robert Boyd, fusilado junto al general Torrijos, tuvo precisamente el honor de estrenar 5 . El irlandés Martin Haverty (1809-1887), autor de Wanderings in Spain in 1843 (1844), daba similares referencias del cónsul Mark y de su meritoria adquisición en 1830 de un terreno destinado a ser el primer cementerio protestante en España, donde, en efecto — también añadía - poco después sería enterrado el militar irlandés Boyd. A partir de entonces los expatriados que residían en Málaga podían ya ser inhumados con dignidad. En 1830 se había conseguido también, tras muchos años de esfuerzo diplomático, que Fernando VII concediera por real orden permiso para construir un cementerio inglés en cada ciudad que tuviera representación consular, que no era poco.

Entre tantas críticas al lamentable estado del país y sus gentes, en su primer volumen de The Shores of the Mediterranean (1837) Standish nos proporciona interesante información sobre la existencia de residentes británicos en el sur de España durante la década de los treinta. Da asimismo a entender que él mismo era un viajero en busca de la salud perdida, es decir, una especie de convaleciente itinerante que aprovechaba sus viajes para obtener, aparte de salud, otras «gratificaciones intelectuales». Como él mismo demuestra, los británicos se muestran ya muy interesados a estas alturas del siglo XIX por conocer las temperaturas, climas y condiciones de salubridad de ciertas ciudades españolas (Málaga, Cádiz, Sevilla), pues datos al respecto, al igual que sobre otros aspectos de utilidad para el

${ }^{5}$ Ford ironiza sobre la ilusión que había demostrado el cónsul Mark de estrenar su cementerio, aunque fuera con su esposa Harriet, convaleciente de frágil salud (Prothero 1905:74-75). No fue sin embargo un convaleciente de frágil salud, como hubiera sido previsible, el que estrenó el cementerio malagueño, sino un «viajero de paso», el mencionado teniente Boyd. 
aspirante a residente, como pueden ser la calidad de los criados españoles o de las carnicerías de cada ciudad —información importante para sí mismo, que sufre de gota— no faltan en ese primer volumen. Ello revela que, en efecto, según Standish, parece ya haber un considerable sector de invalids potenciales con la vista puesta en las ciudades españolas.

Conforme nos acercamos a la mitad del siglo XIX, comenzamos a percibir el creciente número de viajeros de habla inglesa que visitan España por motivos de salud. Entre sus intenciones confesables se encuentra el deseo de pasar en nuestros paradisíacos parajes temporadas de variable duración en aras de mejorar sus delicadas constituciones físicas. Una de los primeras que escribe un relato de viaje por España es Dora Quillinan (1804-1847), autora de Journal of a Few Months' Residence in Portugal, and Glimpses of the South of Spain (1847), hija del poeta William Wordsworth y esposa del también poeta Edward Quillinan. La mayor parte de su estancia de varios meses en el extranjero, entre 1845 y 1846, tuvo lugar en Lisboa, a la sazón ciudad de moda entre los convalecientes del momento, pero no desaprovechó la ocasión de visitar España. Su trayecto por nuestro país fue eminentemente costero y más concretamente mediterráneo (Cádiz, Sevilla, Cádiz, Gibraltar, Málaga, Loja, Granada, Loja, Málaga, Cartagena, Alicante, Valencia y Barcelona), actitud típica del viajero que busca el beneficio de los rayos del sol. Quillinan dedica todo su tiempo libre, que es mucho, a tomar el sol y a dibujar monumentos, hecho que suscita no poca desconfianza entre la población y las fuerzas de seguridad, pues creen que bajo su disfraz de inocente visitante enferma puede ocultarse una espía (1847, II: 215)

En efecto, a mediados de siglo comenzaba a generalizarse el viaje a España por motivos de salud en perjuicio de otros lugares que hasta el momento habían monopolizado las estancias de los viajeros convalecientes: la Riviera franco-italiana, Lisboa, Italia, etc. Incluso Richard Ford, deseoso de abarcar el mayor número de posibles usuarios del influyente y a la sazón imprescindible Handbook for Travellers in Spain, and Readers at Home (1845), decidió incluir en su tercera edición (1855), la última que hiciera en vida, un apéndice titulado «Hints to Invalids» (1855: 37-41) expresamente dirigido a este tipo de viajero. Debido a que Ford, una vez abandonado nuestro país en 1833, no había vuelto a poner el pie en él, se basa esencialmente en los datos recogidos de primera mano y puestos al día por el Dr. Dayrell Joseph Tradwell Francis en su popular obra Change of Climate $(1853)^{7}$. En la obra del médico se describen las excelencias de nuestro clima, y en similares términos se expresa Ford en su «Handbook» de $1855^{8}$. La obra de Francis confirma lo que Ford ya venía anticipando en ediciones anteriores del «Handbook»: que, en efecto, el clima del sur de España era superior al de cualquier otra región de Europa, inclusive Italia, hasta entonces tan de moda para los llamados «travellers expatriated in search of health» (37).

${ }^{6}$ Dora Quillinan falleció al poco tiempo de regresar a Inglaterra de su viaje por España y Portugal.

${ }^{7}$ El título completo de la obra del Dr. Francis es el siguiente: Change of Climate considered as a remedy in dyspeptic, pulmonary, and other chronic affections; with an account of the most eligible places of residence for invalids in Spain, Portugal, Algeria, etc. at different seasons of the year; and an appendix on the mineral springs of the Pyrenees, Vichy, and Aix les Bains. [Etc] (London: John Churchill) (1853).

${ }^{8}$ Ford en cambio titula el libro del Dr. Francis de la siguiente guisa: Change of Climate \&c., with an account of the most eligible places of residence for invalids in Spain, Portugal, Algeria, \&c. By D. J. T. Francis, M. D. (London, 1853) (Ford, 1855: 37). 
Las costas españolas comenzaron a tomar peso como destino apropiado para la convalecencia gracias también a que se extendió en el mundo anglosajón la creencia, sin duda exagerada, de que en las restantes regiones del sur de Europa así como en el norte de África las fiebres, el mal olor y la insalubridad estaban peligrosamente extendidas (Pemble, 1988:240-255). A ello contribuyeron también dos factores más: por un lado, el supino desconocimiento que existía en la época -inclusive en la propia clase médicasobre enfermedades como el tifus o la malaria, conocidas popularmente como «la fiebre romana», supuestamente abundante en Roma, o como «la fiebre de Jericó», llamada así porque se creía procedente de Tierra Santa; por otro lado, la psicosis generalizada que sentían los médicos británicos victorianos por la temible y temida tuberculosis, tal y como demuestran en sus relatos de viajes por el Mediterráneo (241). Aunque algún que otro viajero-médico de la época (como James Henry Bennet, 1875) llegó incluso en alguna ocasión a señalar con el dedo a Málaga como lugar que debían también evitar los convalecientes extranjeros, la realidad es que la mayoría de los facultativos de la época atribuían a ciudades como Roma, Milán o Nápoles o El Cairo, a determinadas zonas costeras como la occidental italiana, la Riviera franco-italiana y el sur de Italia, Sicilia, las islas griegas o Anatolia, o a países enteros como Argelia, Túnez o Malta, un a todas luces exagerado índice de mortandad entre invalids debido a las pésimas condiciones sanitarias del literal mediterráneo (242-45). Curiosamente las costas españolas se salvaron de recibir críticas excesivamente virulentas al respecto; si acaso, todo lo contrario. Salvo alguna excepción, abundaron las opiniones abiertamente favorables al clima y salubridad de nuestro litoral. En consecuencia, junto a los Alpes suizos y otros destinos invernales de moda del momento, por ejemplo, el norte de Egipto, España y sus costas cobraron a partir de mediados de siglo una considerable popularidad entre los viajeros de habla inglesa en busca de la salud perdida.

El citado Dr. Francis hizo en su Change of Climate un estudio pormenorizado de las distintas ciudades españolas y su validez como lugares de convalecencia para el invalid y el «consumptive patient», las cuales había visitado él personalmente entre 1848 y 1849. Naturalmente se dejó influir por las opiniones de los cónsules británicos destinados en ellas (Mark en Málaga, Williams en Sevilla, etc), de los mismos residentes extranjeros que conoció en su periplo español, de otros colegas médicos suyos, y sobre todo de la segunda edición del «Handbook» de Ford, compatriota al que alaba constantemente. Lo califica, dice, de «the great modern observer of Spanish things» (1853: 138), o se refiere a él como «that highly gifted and keen observant» (233).

En Change of Climate Francis recomienda Málaga para las dolencias pulmonares; para los que sufren de digestiones pesadas, sugiere los Pirineos; para las afecciones nerviosas y urinarias, Granada; Lisboa para la lepra; Cádiz para las enfermedades coronarias; Alicante para las dolencias de pecho, etc., y así numerosas otras ciudades de nuestra geografía, como Tarragona y Barcelona, e inclusive el norte del país: Vigo, La Coruña, Gijón, Santander o San Sebastián, más alejadas de las más transitadas y populares rutas mediterráneas, lo que resulta novedoso. Francis es el gran cantor de las excelencias del clima español para el enfermo anglosajón; sobre todo Málaga, que goza, según él, de «the mildest climate in Europe» (1853: 165). Entre las recomendaciones comunes a todos los viajeros por España por motivos de salud, sugiere evitar Gibraltar y el brasero, pero insiste en la conveniencia de montar a caballo con frecuencia (91-92). 
La influencia del libro de Francis se hace más que evidente en el capítulo «Hints to Invalids» de la tercera edición del «Handbook» de Ford (1855). En efecto, de toda la Península Ibérica, también Ford se inclina por Málaga como «the most favoured winter residence in Europe» (1855: 37), a pesar de tener escasas atracciones para el convaleciente. Una de ellas es sin embargo el recién creado cementerio protestante de Mark. Esta ciudad, sigue diciendo, es por lo tanto ideal para el aburrimiento. El vacío mental y cultural que provoca Málaga en el convaleciente que allí reside resulta de lo más beneficioso para su salud: «At Malaga the invalid leads a quiet life, calm as the climate, and, blessed with an otiose oriental real dolce-far niente existence, can leave nature to her full vis medicatrix» (38). Al igual que hiciera Francis, Ford incluye otras zonas costeras del noroeste de España o la costa mediterránea en su totalidad, desde Barcelona a Cádiz. España, escribe Ford, son ideales para ciertos tipos de convalecientes: para los que sufren de consumición, dispepsia, bronquitis y dolencias crónicas (37). Las ventajas de la dieta mediterránea son ya perceptibles para un viajero experimentado como Ford: el agua es pura, el vino barato y de calidad; la dieta española, basada en el aceite, el vinagre y las verduras, tienen evidentes cualidades para el que las consume con asiduidad, dando vida al que tan necesitado está de ella: y si no —añade el viajero no sin cierta ironía— obsérvese el excelente estado de salud de los españoles:

The sustaining effect [of an oil, vinegar, and vegetable diet] is proved by the untiring activity of the very under-fed masses, where many seem to live on air, like chameleons. How strong are Spanish lungs — teste their songs — and how few are their winter-coughs - teste their churches! (38).

Ford resume las actividades del invalid en tomar el aire, ignorar al máximo las medicinas prescritas por los médicos y, en definitiva, limitarse a llevar un ritmo de vida de relax absoluto. Como medida preventiva de posibles enfermedades y dolencias, sugiere también realizar «a riding tour in Spain» (38), consejo que, recuérdese, también diera Francis (1853: 91). Ford sabe por experiencia propia —nostálgico quizás de sus ya lejanas «wild and joyous rides through tawny Spain» (1855: 39-40)—, que ésta es una actividad de lo más reconstituyente y beneficiosa para el cuerpo y el espíritu. Incluye además un generoso listado de balnearios españoles, como también hacía Francis, si bien critica la mayor parte de sus instalaciones, a las que califica de «rude, inadequate, and inconvenient» (40).

A partir de la segunda mitad del siglo XIX se comienza a generalizar la consideración de España como no sólo una más de las muchas zonas del Mediterráneo destinadas a la residencia invernal de los invalids británicos, sino como una de las mejores. Ahora toman el protagonismo los médicos-viajeros. Algunos de ellos se dedican a escribir relatos donde priman aspectos como la observación del clima de la zona, su flora y vegetación, el número de horas de sol y lluvia que recibe una comarca o ciudad determinada, el análisis de su geología o la calidad de sus instalaciones sanitarias.

El mencionado Dr. Francis, «the Clark of Spain» en palabras de Ford (37), fue, como hemos visto, uno de los primeros, mas no es el único. Un médico llamado Edwin Lee, especializado en dolencias pulmonares y la incidencia del clima en ellas, había ya escrito varias obras sobre Niza, las costas francesas e italianas o las excelencias de los balnearios 
alemanes e ingleses antes de publicar Notes on Spain; with a Special Account of Malaga and its Climate (1854)9 , sólo un año después de la del Dr. Francis. En su obra, Lee analiza la disponibilidad y adecuación sanitaria de ciudades como Barcelona, Valencia, Alicante, Elche, Murcia, Cartagena, Almería, Cádiz, Sevilla, Córdoba y Madrid y, sobre todo, Málaga a la que dedica dos capítulos en exclusividad. Las desventajas de las ciudades españolas, asegura, son sin embargo tanto su lejanía de Inglaterra como su difícil acceso por tierra (1854: iv-v). Lee dedica numerosas páginas a asuntos de interés para el convaleciente tales como el número de horas de sol que recibe cada una de estas ciudades, y con especial interés a las temperaturas medias de Málaga durante los años 1849, 1850 y 1851, datos que obtiene de Topografía Médica (1852) de Vicente Giráldez, médico del hospital militar de Málaga que sabía hablar inglés (Krauel Heredia, 1988:135). Sin embargo, de Málaga Lee critica sobre todo la falta de alojamiento adecuado, la inexistencia de chimeneas o estufas en las casas y el excesivo uso del peligroso brasero, que emana humos perjudiciales para el enfermo (1854: 54-55). También muestra preocupación por la salud religiosa de sus compatriotas. Según Lee, gracias al cónsul Mark y al reverendo Charles Brereton ${ }^{10}$, un asmático curado en Málaga, los invalids británicos que se decantaran por dirigirse a esta ciudad no deberían preocuparse pues tendrían plenamente resueltas sus necesidades espirituales. Para ello Mark había aportado un salón contiguo al consulado para los servicios religiosos con una capacidad de cien personas y Brereton los oficiaba (56-58).

Uno de los médicos-viajeros británicos más influyentes entre sus compatriotas es James Henry Bennet, autor de Winter and Spring on the Shores of the Mediterranean: or, the Riviera, Mentone, Italy, Corsica, Sicily, Algeria, Spain, and Biarritz, as Winter Climates $(1870)^{11}$. Bennet llevaba veinte y cinco años de ejercicio como médico cuando se

${ }^{9}$ Prueba del éxito comercial de la obra es su reedición con otro título sólo un año después: Spain and its Climate: with a Special Account of Malaga (London: W. J. Adams, 1855). Fue además reimpresa en 1860.

${ }^{10}$ Charles Brereton, ministro de la iglesia anglicana y particular médico del espíritu, llevaba cinco años como capellán de la comunidad británica en Málaga cuando publicó Lectures on the Christian Faith and Life addressed to the congregation of the Church of England at Malaga (1854), colección de sermones pronunciados desde 1852 a 1854 para las variadas tipologías de visitantes anglófonos que el reverendo observa visitaron su templo en calidad de parroquianos. Se trata de un considerable número de residentes temporales, visitantes ocasionales, viajeros de paso, marineros y mecánicos así como residentes ingleses permanentes (1854:v). La misión de Brereton, así lo percibe, es doble: por un lado, luchar contra la idea generalizada entre los españoles (y en general los habitantes de cualquier lugar del continente) de que «inglés» era casi sinónimo de moro, judío, infiel y pagano (v y ix); por otro, atender espiritualmente a los muchos invalids venidos hasta aquí por el clima, así como a sus acompañantes (v). Brereton lamenta que el orgullo que sienten sus compatriotas por su patria y por su condición de británicos dondequiera que se encuentran — «Civis Romanus sum»— no se corresponda con un similar orgullo por ser y por querer demostrar su condición de cristianos, sobre todo, añade, el religioso, teniendo en cuenta que las diferentes naciones del mundo los toman a ellos, los británicos, como ejemplo a imitar (x). Al igual que Dios quiso que Roma fuera el centro del mundo conocido, sigue diciendo, así debería ser Inglaterra, incuestionable centro del comercio mundial y cuna de la raza más activa y energética y portadora tanto de la civilización y las mejoras tecnológicas; debería asimismo ser la principal divulgadora del mensaje de vida eterna del cristianismo a todos los rincones del planeta (xi-xii). Para más detalles sobre la presencia de viajeros-religiosos durante el siglo XIX, consúltese Ruiz Mas (2005).

${ }^{11}$ Existe una edición posterior de la obra, aumentada y con distinto título: Winter and Spring on the Shores of the Mediterranean: or, the Genoese Rivieras, Italy, Spain, Corfu, Greece, the Archipelago, Constantinople, Corsica, Sicily, Sardinia, Malta, Algeria, Tunis, Smyrna, Asia Minor, with Biarritz and Arcachon, as Winter Climates (London: J. \& A. Churchill, 1875). 
le diagnosticó tuberculosis. Pasó entonces diez años como convaleciente en Mentone (en la Riviera francesa), desde 1859 a 1869 , y al curarse decidió dedicarse a viajar por las costas del Mediterráneo con la intención de encontrar el clima más favorable para los enfermos. Para Bennet (1870: viii), cabe diferenciar dos tipos de climas invernales: el suave y seco, propio de la Riviera y la costa este de España, y el suave y húmedo, característico de Córcega, Sicilia y Argelia. Su informe sobre la conveniencia de ciudades costeras españolas como Barcelona, Alicante o Valencia para el enfermo extranjero no es en general favorable, por ser cada una de ellas, dice, importantes puertos comerciales. Ha de admitir, empero, que gozan todas de excelente clima:

They are not health cities, but social and commercial centres in which invalids and sick people are not thought of. Health towns, such as Cheltenham, Turnbridge Wells, Torquay, Pau, Nice, and Mentone do not exist in Spain. Thus although the winter climate is excellent in some of these [Spanish] cities, real invalids cannot comfortably or prudently remain because there is no provision for them (553).

Sevilla y Córdoba quedan, a juicio de Bennet, descartadas del «grand tour invalid» por ser ciudades eminentemente turísticas y de vegetación poco recomendable para el convaleciente (562). Igual le ocurre a Granada, que tiene además inviernos muy fríos, frecuentemente con nieve, y veranos excesivamente cálidos (570) si bien, admite que sería la más apropiada para residencias estrictamente primaverales y otoñales (572). De Málaga se decepciona, a pesar de su creciente popularidad como lugar de residencia invernal. Tiene, dice, un sucio puerto comercial, demasiado pequeño para una ciudad tan populosa de ciento diez mil habitantes; toda su población vive concentrada en estrechas calles; sus playas están contaminadas y sus hoteles son pobres y lúgubres. Málaga sólo puede ser lugar de residencia para «the poor invalids condemned for their sins to winter here» (566). Añade con ironía que «the only real garden within three miles of the town is the English cemetery (...)» (566) ${ }^{12}$. Para Bennet, las costas españolas en general no pueden compararse a las de la Riviera. España, insiste, no está preparada para recibir al convaleciente extranjero, y no lo estará hasta que se realicen en ellas las necesarias adaptaciones:

[The invalid's] choice can only lie between the Genoese undercliff and the South-east of Spain. At the same time, in my opinion, real invalids are not, at present, warranted in choosing any part of Spain as a winter residence, from the want of any kind of preparation to receive them. The only hotels are commercial hotels in the midst of commercial towns, and there are no villas out of the towns. The Genoese Riviera, on the other hand, being fully prepared to receive invalids, it must have the preference until such time as Spanish or foreign capitalists make the necessary preparations for them in Spain (580-81).

Bennet nos relata en su obra una interesante visita que hizo a un ilustre expatriate británico residente en las afueras de Murcia. Se trataba del ex-embajador británico en

12 Sobre las distintas opiniones vertidas en los libros de viajes en lengua inglesa durante el siglo XIX sobre una ciudad tan característica del Mediterráneo español como Málaga, véase López-Burgos del Barrio (2006). 
Madrid, Lord Howden ${ }^{13}$. La casa y el fértil solar que éste había adquirido doce años antes es un verdadero vergel de plantas y flores que el visitante, gran entendido en botánica, describe con gran generosidad de detalles (534-35). El clima, añade Bennet, es la causa de la frondosidad de un jardín así y en general la de los jardines españoles, que raramente falta en la casa de un expatriado británico que se precie: «[The garden] is very interesting as an evidence of the rapidity of growth in such a climate, with rich earth and water ad libitum, if what his bailiff told me be correct». (534).

Algunos ciudadanos norteamericanos de la época muestran asimismo cierto interés por encontrar zonas ideales del Mediterráneo donde poder pasar las convalecencias invernales. Prueba de ello es la obra del embajador y senador de Nueva York Samuel Sullivan Cox (1824-1889), Search for Winter Sunbeams in the Riviera, Corsica, Algiers, and Spain (1870). La ruta de Cox por España es eminentemente costera (Valencia, Alicante, Elche, Murcia, Cartagena, Granada y Málaga), pero no por ello excluye una posible ruta interior que pasaría por Sevilla, Córdoba, Toledo, Madrid y alrededores y Zaragoza. Aunque con altibajos, se podría ya hablar de cierta institucionalización de la presencia de convalecientes de habla inglesa en España, en la que se incluye ya a algún norteamericano, durante el último tercio del siglo XIX.

Finalmente, cuando el teniente coronel R. L. Playfair viaja por España, a la que dedica sólo un breve capítulo de sus dos volúmenes del Handbook to the Mediterranean: its Cities, Coasts, and Islands (1881), lo hace casi exclusivamente por su costa oriental: Barcelona, Tarragona, Valencia, Alicante, Elche, Cartagena, Almería, Málaga, desde donde se traslada a Granada y Córdoba y Gibraltar. Playfair (1881, II: 443) dedica los más encendidos elogios a las islas Baleares, parabienes que coinciden plenamente con los de Bidwell. Sin embargo, Eustace A. Reynolds Ball, autor de Mediterranean Winter Resorts: a Practical Handbook to the Principal Health and Pleasure Resorts on the Shores of the Mediterranean (1888), prefiere dedicar toda la parte II — de cinco- a Málaga y a Gibraltar.

En este breve recorrido por la imagen de la España mediterránea durante el siglo XIX que transmitieron en sus relatos de viaje los médicos y convalecientes de habla inglesa que buscaron en nuestras templadas ciudades y costas la cura o la mejora de sus delicadas saludes, hemos podido apreciar cómo los lugares tradicionalmente favorecidos como Italia, Francia y Portugal iban dejando paso a España conforme se llegaba a conocer mejor nuestro país a lo largo del siglo. Los primeros enfermos y convalecientes, aún no excesivamente numerosos, se encontraron durante el primer tercio del siglo con una coyuntura histórica — las desamortizaciones eclesiásticas y el caos posterior a la Guerra de la Independencia — que les permitió adquirir a muchos de ellos obras pictóricas de gran valor artístico. A partir de la segunda mitad de siglo se incrementó espectacularmente el número de visitantes gracias a la labor difusora de varios médicos-viajeros anglófonos que promocionaron los valores terapéuticos del sur y del este español, in-

${ }_{13}$ El barón Sir John Hubart Caradoc (Dublín, 1799-Bayona, 1873), segundo Lord Howden, fue militar, diplomático y ministro plenipotenciario en Madrid desde 1850 a 1858. Gozó de gran popularidad entre los gobernantes y ciudadanos españoles por su buen carácter y especialmente por comparación con su predecesor Sir Henry Bulwer, expulsado por Narváez por entrometerse demasiado en los asuntos de estado españoles. Lord Howden se retiró sin derecho a pensión por problemas de salud (The Dictionary of National Biography, 1973, III:938). 
clusive sus islas - Canarias y Baleares - debido a sus climas soleados y a la carencia de fiebres y otras enfermedades que se prodigaban, por el contrario, en las costas de otros países del Mediterráneo.

\section{OBRAS CITADAS}

\section{Fuentes primarias}

Ball, E. A. Reynolds (1888): Mediterranean Winter Resorts: a Practical Handbook to the Principal Health and Pleasure Resorts on the Shores of the Mediterranean. By ... With 27Illustrations, mostly from Sketches taken on the Spot by C. W. J. Praetorius, and a Sketch-Map of the Mediterranean. London: L. Upcott Gill.

BENNET, J. Henry (1870): Winter and Spring on the Shores of the Mediterranean: or, the Riviera, Mentone, Italy, Corsica, Sicily, Algeria, Spain, and Biarritz, as Winter Climates. By ... M.D. [Etc]. Fourth Edition. London: John Churchill \& Sons.

BIDwell, Charles Toll (1876): The Balearic Islands. By ... F.R.G.S. With Maps and Illustrations. London: Sampson Low, Marston, Searle and Rivington.

BRERETON, Charles (1854): Lectures on the Christian Faith and Life Addressed to the Congregation of the Church of England at Malaga. By the Chaplain, the Reverend ..., M.A.; of Trinity College, Cambridge; Late Rector of St. Edmund's, Norwich; Formerly an Assistant-Curate in St. James's, Westminster. London: Thomas Bosworth.

Cox, Samuel Sullivan (1870): Search for Winter Sunbeams in the Riviera, Corsica, Algiers, and Spain. With Numerous Illustrations. By S. S. Cox. New York: C. Appleton \& Company.

FoRD, Richard (1845): A Handbook for Travellers in Spain, and Readers at Home. Describing the Country and Cities, the Natives and their Manners; the Antiquities, Religion, Legends, Fine Arts, Literature, Sports, and Gastronomy: with Notices on Spanish History. [Parts I and II]. London: John Murray.

- (1855): A Handbook for Travellers in Spain. By ..., F.S.A. [Parts I and II, etc]; The Portions Best Suited for the Invalid - A Winter Tour. Quien Dice España - Dice Todo. Third Edition, Entirely Revised, with Great Additions. London: John Murray.

FRANCIS, D. J. T. (1853): Change of Climate Considered as a Remedy in Dyspectic, Pulmonary, and Other Chronic Affections; with an Account of the most Eligible Places of Residence for Invalids in Spain, Portugal, Algeria, etc., at Different Seasons of the Year; and an Appendix on the Mineral Springs of the Pyrenees, Vichy, and Aix Les Bains. By ..., M.D. Lond. Corresponding Member of the Society of the Medical Sciences of Lisbon, of the Academy of Medicine and Surgery of Barcelona, \&c.; Physician to the Dispensary for Consumption and Diseases of the Chest, Margaret Street, Cavendish Square. [Etc]. London: John Churchill.

HAVERTY, Martin (1844): Wanderings in Spain in 1843. London: T. C. Newby. 2 vols.

[Holland, Lady] (1910): The Spanish Journal of Lady Elizabeth Holland. Edited by the Earl of Ilchester. With Portrait and Map. London, New York, Bombay, and Calcutta: Longmans, Green and Co.

LeE, Edwin (1854): Notes on Spain; with a Special Account of Malaga and its Climate. By E. Lee. London: Hope \& Co.

PlaYfaIr, Lieut.-Col. R. L. (1881): Handbook to the Mediterranean: its Cities, Coasts, and Islands. For the Use of General Travellers and Yachtsmen. By ... With Many Maps, Plans, \&c. London: John Murray.

Quillinan, Dorothy (1847): Journal of a Few Months' Residence in Portugal, and Glimpses of the South of Spain. London: Edward Moxon. 2 vols. 
Standish, F. H. (1832): The Maid of Jaen, a Poem. With Notes and General Remarks. [Etc]. By ..., Esq. New Edition. Chorley: C. Robinson.

- (1837-38): The Shores of the Mediterranean. By ... Esq. Vol. I: London: Edgard Lumley; Vol. II: London: Black and Armstrong.

\section{Fuentes secundarias}

Alberich, José (1976): Del Támesis al Guadalquivir. Antología de viajeros ingleses en la Sevilla del siglo XIX. Sevilla: Secretariado de Publicaciones de la Universidad de Sevilla.

- (2001): El cateto y el milor y otros ensayos angloespañoles. Sevilla: Universidad de Sevilla.

BEAN, Thomas (1995): «Richard Ford as Picture Collector and Patron in Spain». Burlington Magazine, February 1995. 96-107.

FORD, Brinsley (1974): «Catalogue». A Loan Exhibition. Richard Ford in Spain in Aid of the National Collections Fund $5^{\text {th }}$ July - $12^{\text {th }}$ July, 1974. London: Wildenstein. 45-90.

Krauel Heredia, Blanca (1986): Viajeros británicos en Andalucía de Christopher Hervey a Richard Ford (1760-1845). Málaga: Universidad de Málaga.

LóPEZ-Burgos Del BARRIO, M. Antonia (2006): «A Disappointing Tale of Two Cities: Málaga and Almería in XIXth Century English Travel Literature». En The English Lake. British Travellers in the Mediterranean, de López-Burgos del Barrio, M. A. and Ruiz Mas, J. (eds). Granada: Editorial Universidad de Granada. 39-54.

Pemble, John (1988): The Mediterranean Passion. Victorians and Edwardians in the South. Oxford, New York: Oxford University Press.

PROTHERO, Rowland E. (Ed.) (1905): The Letters of Richard Ford 1797-1858. Edited by ... M.V.O. Formerly Fellow of All Souls' College, Oxford, Author of «The Life of Dean Stanley», «The Psalms of Human Life», Etc, Etc. With Portraits and Illustrations. London: John Murray.

RuIZ MAs, José (2005): «El antipapismo de los viajeros-religiosos por la España de mediados del siglo XIX». En Viajeros británicos, irlandeses y norteamericanos en España: escritores, pintores y músicos, de López-Burgos del Barrio, M. A. y Ruiz Mas, J. (eds). Granada: Editorial Universidad de Granada. 254-264.

- (2006): Sibaritas al sol. Aventuras y desventuras de los residentes de habla inglesa en España. Granada: Grupo Editorial Universitario y Centro Asociado de la UNED de la Provincia de Jaén. 